\title{
Estudio piloto: eficacia de una intervención en inteligencia emocional en universitarios con sintomatología depresiva
}

\section{Pilot study: effectiveness of an intervention in emotional intelligence in university with depressive symptomatology}

Patricia Alejandra Rivera-Suárez

Manuel Sosa-Correa

Universidad Autónoma de Yucatán
Sally Vanega-Romero

Antonio Zayas

Universidad de Cádiz

\section{Resumen}

La relación entre Inteligencia Emocional (IE) y depresión es muy estrecha, las personas depresivas prestan excesiva atención a sus pensamientos, tienen poco claras sus emociones y no saben regularlas de forma adecuada (Mestre, \& Fernández-Berrocal, 2009). La depresión puede considerarse como un trastorno de la regulación del estado de ánimo, por ello, el objetivo principal de este trabajo es evaluar la eficacia de una intervención psicológica dirigida a fomentar las capacidades en la regulación emocional y conocer su efecto en la sintomatología depresiva. Para ello, se implementó un taller de integración técnica de orientación, que incluía técnicas de la Terapia Racional Emotiva Conductual, que además fueran congruentes con los objetivos y estrategias del modelo de las cuatro ramas de Salovey y Mayer (Sosa-Correa et al, 2015). La muestra estuvo conformada por doce universitarios, con edades entre 19 y 34 años $(M=22.5$ y $D E=4.54)$, con síntomas de depresión. Se administraron dos instrumentos, la Escala Autoinformada de Inteligencia Emocional de Correa, el Inventario de Depresión de Beck-II, así como una evaluación de la sesión. Los resultados muestran que el taller tuvo efecto positivo significativo en el aumento de ocho de las once subescalas de la prueba de IE, y en seis participantes disminuyeron los síntomas depresivos.

Palabras clave: emocional (IE), terapia racional emotiva, depresión, enfoque integrativo.

Nota del autor

Patricia Alejandra Rivera-Suárez, Universidad Autónoma de Yucatán (UADY), Carr. Mérida-Tizimín, km 1, Cholul, Yucatán, C. P. 97305. Celular: 9995753261, correo electrónico: mscorrea@correo.uady. mx Manuel Sosa-Correa, Universidad Autónoma de Yucatán. Sally Vanega-Romero, Universidad de Cádiz. Antonio Zayas, Universidad de Cádiz. 


\begin{abstract}
Emotional intelligence (IE) and depression demonstrate a close relationship, where those diagnosed with depression pay an inordinate amount of attention to their thoughts, are unclear about their emotions and do not know how to regulate them properly (Mestre, \& Fernández-Berrocal, 2009). Depression can be considered as a lack of mood regulation disorder. Thus, the main objective of this study was to evaluate the effectiveness of a psychological intervention aimed at building emotional regulation abilities and explore its effect on depressive symptomatology. A technical orientation integration workshop was used, which included Rational Emotive Behavioral Therapy techniques, congruent with the objectives and strategies of the Salovey and Mayer four branches model (SosaCorrea et al, 2015). The sample included 12 university students, ages ranging from 19 to $34(M=$ 22.5 and $S D=4.54)$, who had previously reported depression symptoms. To measure the effects of the workshop, two instruments were used, the Self-Informed Emotional Intelligence Scale (EAIE, Sosa-Correa, 2008) and the Beck Depression Inventory-II (BDI-II, Beck; Steer, \& Brown, 2009) and sessions were evaluated. Results show that the workshop had a significant positive effect, increasing 8 of the 11 subscales of the IE test, and there was a decrease in depressive symptoms in 6 participants.

Keywords: emotional intelligence, rational emotive behavioral therapy, depression, integrative approach.
\end{abstract}

El concepto de Inteligencia Emocional (en adelante IE), fue desarrollado por Salovey y Mayer (1990), como un tipo de inteligencia social que incluye una habilidad de supervisar y entender las emociones propias y las de los demás, discriminar entre ellas y usar la información para guiar los pensamientos y las emociones en uno, que incluye la evaluación y expresión verbal y no verbal de las mismas, la regulación y utilización de la información emocional (Sosa-Correa, 2008).

Actualmente, se cuenta con dos grandes modelos de IE; los modelos mixtos, representados fundamentalmente por Bar-On (1997) y Goleman (2009), quienes combinan dimensiones de personalidad, como asertividad u optimismo, con habilidades emocionales; y los modelos de capacidades, que conciben a la IE como una inteligencia genuina basada en "el uso adaptativo de las emociones en la cognición de forma que el individuo pueda resolver problemas y adaptarse eficazmente al medio" (Sánchez-González, 2014, p. 12), representando por Salovey y Mayer en 1990.

Posteriormente, Mayer y Salovey en 1997, dieron mayor énfasis a los aspectos cognitivos reorganizando la IE en cuatro ramas: percepción, evaluación y expresión de las emociones; Facilitación emocional del pensamiento; Entendimiento y análisis de las emociones; Empleo del conocimiento personal; y La regulación de las emociones para promover el crecimiento emocional e intelectual (Mestre, \& FernándezBerrocal, 2009). 


\section{Depresión}

La depresión es una alteración del estado emocional que abarca síntomas fisiológicos, conductuales, cognitivos, emocionales o intelectuales, entre otros. Presenta una gran incidencia y prevalencia en la población general, mermando la calidad de vida y provocando mayor morbimortalidad, repercusiones sociofamiliares, laborales, conyugales, altos costos directos e indirectos (Chinchilla-Moreno, 2008).

Se produce cuando los sentimientos de profunda tristeza o desesperación duran como mínimo dos semanas y cuando interfieren con las actividades de la vida cotidiana. Las personas deprimidas tienden a sentirse indefensas, sin esperanzas, culpables por padecer esta sintomatología, llegando, a tener pensamientos suicidas (American Psychological Association [APA], 2016).

Según la teoría de Beck, la depresión se caracteriza fundamentalmente por la existencia de errores cognitivos que conducen al individuo deprimido a percibir e interpretar la realidad de una manera negativa, caracterizándose por un procesamiento distorsionado y de forma negativa de la realidad (Ortiz, 1997).

\section{Depresión en el adulto joven}

Para Papalia, Sterns, Duskin y Camp (2009), los adultos jóvenes se caracterizan por tres tipos de esferas: 1. El desarrollo físico: se encuentran en la cima de su condición física, las elecciones del estilo de vida influyen en la salud. 2. El desarrollo cognitivo: las habilidades cognitivas y los juicios morales implican una mayor complejidad y 3. El desarrollo psicosocial: los rasgos de personalidad y los estilos de vida se vuelven relativamente estables.

En esta etapa se da una serie de transformaciones que puede afectar su salud mental, ya que en la transición de la adolescencia a la juventud aparecen nuevas formas de pensar, sentir y actuar (Arieti, \& Bemporad, 1993).

Chinchilla Moreno (2008) hace referencia a una notable tendencia a la disforia e irritabilidad, acentuación de la impulsividad característica de este periodo evolutivo de la adolescencia a la adultez joven. De igual manera, aumenta el riesgo de fracaso escolar, aparece desinterés por las actividades lúdicas y una disminución de los niveles de motivación intrínseca y extrínseca y autoestima, aumentando los sentimientos de culpa y de inferioridad con respecto al grupo de iguales. Asimismo, se suele dar una afectación en el sueño y la conducta alimentaria, siendo muy característico la aparición de hipersomnia e hiperfagia. Estos síntomas de depresión, así como las ideas recurrentes de muerte aumentan de manera considerable el riesgo de suicidio, por tanto, es de vital importancia prestarle atención científica a esta problemática.

\section{Relación entre la IE y la depresión}

Se ha observado que las personas depresivas prestan excesiva atención a sus pensamientos, tienen poco claras sus emociones y no saben regularlas de forma adecuada (Mestre, FernándezBerrocal, 2009). La relación conceptual entre 
regulación afectiva y depresión es muy estrecha. Tal es así que la depresión podría ser denominada no solo un trastorno del estado de ánimo, sino específicamente un trastorno de la regulación del estado de ánimo, ya que tiene un componente muy importante de descontrol afectivo, o dicho de otra forma, de ruptura de la homeostasis afectiva en la que cada individuo fluctúa.

De ahí que, uno de los conceptos que últimamente ha cobrado relevancia en la psicología, es el modelo de IE de Salovey y Mayer de 1997. Dichos autores argumentan que conseguir un nivel IE adecuado, mejora la comunicación, las relaciones personales, la relación con uno mismo, y especialmente ayuda a la salud, previniendo la depresión. La IE hace referencia a los procesos implicados en el reconocimiento, uso, comprensión y manejo de los estados emocionales de uno mismo y de los otros para resolver problemas y regular la conducta (Sosa-Correa, Navarrete-Centeno, \& Escoffié-Aguilar, 2014).

En recientes estudios, se ha demostrado que la IE permite disminuir la intensidad y la frecuencia de los estados de ánimo negativos provocados por los acontecimientos adversos de la vida cotidiana (Fernández-Berrocal, \& Extremera, 2005). Personas que tienen la flexibilidad y habilidad de confrontar asuntos internos emocionales no sufren de ese desorden. En este sentido, la IE alta protege o hace menos vulnerable al afecto negativo de la depresión, por consiguiente, el bienestar psicológico de la persona aumenta.

\section{La Terapia Racional Emotiva Conductual de} Albert Ellis

La Terapia Racional Emotiva Conductual (en adelante TREC), se basa en la idea de que las emociones como conductas están influidas por las creencias de la persona, es decir, de su interpretación de la realidad (Lega, Caballo, \& Ellis, 2009). Su objetivo es asistir al paciente en la identificación de pensamientos irracionales o disfuncionales, ayudarle a que sea capaz de reemplazar dichos pensamientos por otros más adaptativos para sus propios fines, lo cual permite al individuo lograr con más eficacia metas de tipo personal (Fernández, García, \& Crespo, 2012), empleando métodos que faciliten a la persona reconocer sus creencias irracionales y cambiarlas.

Las emociones adaptativas producto de las cogniciones racionales, reflejan más la IE que las emociones no adaptativas, producto de las creencias irracionales (Spörrle, Welpe, \& Försterling, 2006).

También como parte de la teoría de Ellis (2000), que se incluyó en esta intervención, es la autoaceptación positiva incondicional, es decir: aceptarse como bueno, valioso, o digno de vivir y disfrutar simplemente porque usted es un ser humano, está vivo, y es una persona única y no evaluar o medir su ego o persona, sino solo sus pensamientos, sentimientos, y conductas individuales, centrándose en sus metas y propósitos elegidos (p. 170). 
Para concluir, Ellis (2000) expone que se ha demostrado que la aceptación, disminuye sentimientos de ansiedad o depresión.

Teniendo en cuenta todo lo anteriormente expuesto, nuestro objetivo con el presente trabajo es diseñar, implementar y evaluar un programa de intervención terapéutica que permita aumentar la IE y disminuir los síntomas de la depresión en los universitarios, mediante una intervención basada en las habilidades en IE y la TREC.

\section{Método}

\section{Participantes}

Para la evaluación diagnóstica se realizó un muestreo no probabilístico intencional, al cual acudieron 21 personas para este programa piloto de intervención terapéutica, sin embargo, un criterio de inclusión para formar parte de este estudio, era estar presente en el pretest y el postest, así como acudir como mínimo a $75 \%$ de las sesiones, por ello, solo se tomaron en cuenta las evaluaciones de doce personas, de las cuales nueve fueron mujeres, el rango de edades estuvo entre 19 y 34 años $(M=22.5$ y $D E=4.54)$, que presentaban sintomatología depresiva.

\section{Procedimiento}

Para llevar a cabo la investigación se realizó una revisión a los motivos de consulta de las entrevistas iniciales del Centro de Atención al Estudiante (CAE), que se localiza en los diferentes Campus de la Universidad Autónoma de Yucatán, (en adelante UAYD), así como también se revisó la base de datos, observándose que el motivo de consulta predominante en los años 2014 y 2015 fue la presencia de sintomatología depresiva con $24 \%$ y $27 \%$, respectivamente, de la población que solicitaba el servicio.

Debido a la demanda, se decidió hacer un taller, para lo cual se le solicitó apoyo a la Facultad de Psicología, en lo relacionado con la infraestructura y las instalaciones.

Tabla 1.

Datos sociodemográficos de los participantes

\begin{tabular}{lcc}
\hline Características de la población & Porcentaje de sujetos & Número de sujetos \\
\hline Originarios de Mérida & $83.33 \%$ & 10 \\
Residían en Mérida en el momento de la evaluación & $96.66 \%$ & 11 \\
Estudian la licenciatura & $83.33 \%$ & 10 \\
Estudian posgrado & $16.66 \%$ & 2 \\
Trabajan & $66 \%$ & 8 \\
Reportaron algún problema de salud física & $41.66 \%$ & 5 \\
Reportaron algún problema de salud mental & $33.33 \%$ & 4 \\
Son solteros & $83.33 \%$ & 10 \\
Vive en unión libre & $16 \%$ & 2 \\
Vive con su familia & $91.66 \%$ & 11 \\
Vive solo & $8.33 \%$ & 1 \\
\hline
\end{tabular}




\section{Instrumentos}

Al fin de dar cobertura a nuestro objetivo se aplicaron los siguientes instrumentos:

\section{Escala Autoinformada de Inteligencia Emocional (EAIE, Sosa-Correa, 2008)}

Es una escala autoinformada de tipo Likert con un total de 77 ítems, con opciones del uno al cinco, implicaban que el sujeto se decantara en cada enunciado por estar: 1. Totalmente en desacuerdo, 2. Más en desacuerdo que de acuerdo, 3. Ni de acuerdo ni en desacuerdo, 4. Más de acuerdo que en desacuerdo y 5. Totalmente de acuerdo con una significancia inter ítem de .40 y el Alfa de Cronbach de .982. (Sosa-Correa, 2008). A continuación, se presenta la en la tabla 2, el porcentaje del dominio autopercibido de la persona, según la puntuación reportada.

Los once factores de la EAIE son: factor 1: el darse cuenta, a nivel emocional, tanto en el área intrapersonal como en la interpersonal; factor 2: manejar las emociones, tanto en el área intrapersonal como en la interpersonal; factor 3: predecir el cambio emocional, tanto en el área intrapersonal como en la interpersonal; factor
4: conocer las causas de las emociones y de la expresión emocional en el área interpersonal; factor 5: controlar la distancia emocional en el área intrapersonal; factor 6: controlar la distancia emocional en el área interpersonal; factor 7: conocer la expresión emocional en el área intrapersonal; factor 8: explicar las sensaciones de las emociones, tanto en el área intrapersonal como en el área interpersonal; factor 9: reconocer el cambio emocional, tanto en el área intrapersonal como en el área interpersonal; factor 10: recordar emociones para acordarse de situaciones, tanto en el área intrapersonal como en el área interpersonal; y factor 11: lograr emociones determinadas en el área intrapersonal.

Inventario de Depresión de Beck (BDI-II, Beck; Steer, \& Brown, 2009)

Es un autoinforme que proporciona una medida, tantodela presencia, comodela gravedad de la depresión en adultos y adolescentes de 13 años o más. Se compone de 21 ítems indicativos de sintomatología depresiva.

Tabla 2.

Niveles entre la media de los puntajes y el porcentaje de dominio

\begin{tabular}{cc}
\hline Media del factor & Porcentaje que considera dominar la habilidad del factor \\
\hline $1.0-1.5$ & $0 \%$ \\
$1.5-2.5$ & $25 \%$ \\
$2.5-3.5$ & $50 \%$ \\
$3.5-4.5$ & $75 \%$ \\
$4.5-5.0$ & $100 \%$ \\
\hline
\end{tabular}


Las personas evaluadas han de elegir las afirmaciones que más encajan con su situación personal en las últimas dos semanas. Los ítems son respondidos en una escala de 0 a 3 puntos. Las puntuaciones mínimas y máximas en el test son 0 y 63 . Siguiendo el baremo propuesto por los autores de la prueba, se ha establecido la siguiente clasificación: puntuaciones entre 0-13, depresión mínima; 14-19, depresión leve; 20-28, depresión moderada; y 29-63, depresión severa. El alfa de Cronbach es de 0,92.

\section{Ficha de evaluación de la sesión}

Es una microevaluación escala tipo Likert con cinco opciones que va desde nada a mucho $\mathrm{y}$ que contiene las siguientes tres preguntas respeto a la sesión del taller: ¿qué tanto te gustó?, ¿qué tanto aprendiste?, ¿qué tan útil fue?

\section{Diseño de Intervención}

Al ser un estudio sin grupo control y que busca conocer el efecto de una intervención, se considera un "estudio piloto de efectividad de un solo grupo", según Craig y colaboradores (2008).

\section{Programa de intervención}

Se tomó como punto de referencia teórica la propuesta de intervención de Sosa-Correa y compañeros (2015), integrando el Modelo de IE de Salovey y Mayer (1997) y la TREC (AlbertEllis, 2000), donde se incluyen el desarrollo de las temáticas, emociones primarias; percepción y expresión emocional; autoaceptación; facilitación emocional del pensamiento; facilitación cognitiva de la emoción; comprensión de las emociones y regulación reflexiva de las emociones y prevención de recaídas (Sosa-Correa et al., 2015). Esta integración se clasifica como un "modelo técnico de orientación", ya que selecciona los de procedimientos terapéuticos efectivos, que además sean congruentes con los objetivos de intervención (Rosado y Rosado, Vázquez-Vargas, \& Cetina-Sosa, 2016).

\section{Procedimiento de implementación}

El taller se llevó a cabo durante el mes de enero de 2016, la difusión se hizo mediante folletos y carteles que se divulgaron en las cinco facultades del campus Ciencias Sociales, Económico-Administrativo y Humanidades de la UADY. Para ello, se concertó una cita con los responsables de las áreas correspondientes de cada facultad para los derechos de la difusión y se entregó a cada una cinco folletos y dos carteles que fueron puestos en los tablones de anuncio.

También se contó con el apoyo de la redes sociales que cuenta el CAE difundiendo de esta manera el taller que contenía información sobre el objetivo, horario, lugar, número de sesiones y los datos para contactar con la responsable del proyecto. Por tal motivo, se dejó una lista en CAE para que los interesados anotaran sus datos personales y para ello, se contó con el apoyo de dos estudiantes que realizaban su servicio social en dicha área para el registro de la lista.

La intervención fue un taller de catorce sesiones que incluía el pretest, el postest y las ocho temáticas que se muestran en la tabla 3 : 
Tabla 3.

Modelo integrativo de intervención para el fomento de la IE

\begin{tabular}{|c|c|}
\hline Temas & Objetivo \\
\hline $\begin{array}{l}\text { 1.- Conocimiento de las emociones (Sosa- } \\
\text { Correa, 2009) }\end{array}$ & Conocer la importancia y función de las emociones \\
\hline $\begin{array}{l}\text { 2.- Percepción, valoración y expresión de la } \\
\text { emoción (Salovey, \& Mayer, 1997) }\end{array}$ & $\begin{array}{l}\text { Identificar las emociones propias y las de los demás. } \\
\text { Expresar emociones adecuadamente así como las necesidades } \\
\text { relacionadas con ellas }\end{array}$ \\
\hline $\begin{array}{l}\text { 3.- Facilitación emocional del pensamiento } \\
\text { (Salovey, \& Mayer, 1997) }\end{array}$ & $\begin{array}{l}\text { Priorizar los procesos cognitivos básicos, focalizando nuestra } \\
\text { atención en lo que es efectivamente importante } \\
\text { Lograr sacar ventaja de los cambios de humor y el } \\
\text { afrontamiento diferencial de problemas }\end{array}$ \\
\hline $\begin{array}{l}\text { 4.- Facilitación cognitiva de la emoción. } \\
\text { La influencia de los pensamientos en las } \\
\text { emociones y cómo manejarlas para una } \\
\text { mayor adaptación (Ellis, 1998) }\end{array}$ & $\begin{array}{l}\text { Conocer "cómo nuestros pensamientos afectan nuestras } \\
\text { emociones y conductas". Conocer las herramientas de la } \\
\text { TREC } \\
\text { Conocer qué tipo de pensamientos pueden generar emociones } \\
\text { adaptativas o desadaptativas }\end{array}$ \\
\hline $\begin{array}{l}\text { 5.- Comprensión y analizar las emociones } \\
\text { empleando el conocimiento emocional } \\
\text { (Salovey, \& Mayer, 1998) }\end{array}$ & $\begin{array}{l}\text { Etiquetar las emociones y reconocer en qué categorías se } \\
\text { agrupan los sentimientos } \\
\text { Comprender sentimientos complejos y reconocer las } \\
\text { transiciones de unos estados emocionales a otros así }\end{array}$ \\
\hline 6.- Autoaceptación (Ellis, 1998) & $\begin{array}{l}\text { Identificar las características positivas y negativas de los } \\
\text { participantes }\end{array}$ \\
\hline $\begin{array}{l}\text { 7.- Regulación reflexiva de las emociones } \\
\text { (Salovey, \& Mayer, 1997) }\end{array}$ & $\begin{array}{l}\text { Estar abierto a los sentimientos tanto positivos como } \\
\text { negativos } \\
\text { Monitorizar reflexivamente las emociones en relación con uno } \\
\text { mismo y a otros, así como regular las emociones propias y } \\
\text { ajenas }\end{array}$ \\
\hline 8.- Prevención de recaídas ( Ellis, 1998) & $\begin{array}{l}\text { Informar acerca de la posibilidad de la existencia de una } \\
\text { recaída e identificar los recursos adquiridos en el taller }\end{array}$ \\
\hline
\end{tabular}

La estructura para cada sesión estuvo compuesta por ocho partes, en la primera parte se iniciaba con la toma de temperatura emocional, en donde se le preguntaba a cada uno de los participantes qué emoción estaban sintiendo y con qué intensidad; en la segunda parte se realizaba una actividad con el objetivo de activar y motivar al grupo en la participación de las actividades posteriores. La tercera parte consistía en la revisión de la tarea dejada en la sesión anterior. En la cuarta parte, se procedía a una actividad inductora al tema, en la quinta parte se realizaba una exposición del tema que tocaba para dicha sesión, en la sexta parte para practicar lo explicado en la exposición, es decir, una actividad para integrar la información vista en la sesión. Ya en la séptima parte, se asignaba la tarea para la semana en la que los 
participantes aplicaban en práctica lo visto, y en la octava parte se finalizaba con la aplicación de la microevaluación de la sesión que consistió en una escala tipo Likert que contiene las siguientes tres preguntas: ¿qué tanto te gustó?, ¿qué tanto aprendiste?, ¿qué tan útil fue?, con cinco opciones que van de nada, poco, algo, bastante y mucho, eligiendo una opción para cada pregunta, con el objetivo de evaluar la percepción de los participantes en cuanto al nivel de utilidad, de aprendizaje y de satisfacción. De igual manera, el formato contaba con un espacio para anotar observaciones (sobre los instructores, dinámicas, temáticas u otros).

\section{Evaluación del programa de intervención}

Se trata de un estudio piloto, con una metodología cuantitativa y un diseño preexperimental, el cual, Hernández Sampieri, Fernández Collado y Baptista Lucio (2010) señalan que "a un grupo se le aplica una prueba previa al estímulo o tratamiento experimental, después se le administra el tratamiento y finalmente se le aplica una prueba posterior al estímulo" (p. 136). Para ello, se realizó un muestreo de tipo no probabilístico intencional con mediciones pretest y postest. La evaluación se llevó a cabo en dos fases.

\section{Resultados}

A continuación, se expondrá un análisis descriptivo que compara los resultados obtenidos del pretest y el postest en cada una de las escalas BDI-II y EAIE, seguido por una sección en la cual se describen las pruebas de hipótesis del efecto del taller.

\section{Escala Autoinformada de Inteligencia Emocional (EAIE)}

Como se puede observar en la figura 1, la media de los participantes en cada factor, incrementó en todos, observándose el mayor incremento absoluto en los factores 7 (Conocer la expresión emocional en el área intrapersonal) y 8 (Explicar la sensación de las emociones tanto en el área intrapersonal como en el área interpersonal), y el menor incremento se presenta en factor 2 (Manejar las emociones tanto en el área intrapersonal como en el área interpersonal). De igual forma, se puede observar un incremento de $50 \%$ en el dominio de los factores 4 (Conocer las causas de las emociones y de la expresión emocional en el área interpersonal), 7 y 8 . A excepción del factor 2 , en el cual no se observa un incremento, el dominio del resto de los factores aumentó en $25 \%$.

\section{El inventario de Depresión de Beck (BDI-II)}

A continuación, en la figura 2 se presentan los resultados obtenidos en el BDI-II por los doce sujetos que fueron evaluados en el pretest $\mathrm{y}$ postest, ordenados según su puntuación en el pretest del BDI-II.

Como se puede observar en la figura 2, se registró un decremento en los niveles de depresión en seis sujetos que fueron sometidos a la intervención. Concretamente una persona pasó de un nivel leve a mínimo, dos de moderado a mínimo, uno de moderado a leve, y dos de severo a moderado. 


\section{Resultados de la evaluación de las sesiones}

En la microevaluación de la escala tipo Likert, el puntaje promedio de todas las sesiones fue de 4.45 en relación con la satisfacción con los talleres, 4.21 en relación con el aprendizaje y 4.43 en la utilidad. La sesión en donde se alcanzó el nivel más alto de satisfacción corresponde a la sesión 10 y 11, de aprendizaje, corresponde a la sesión 12 y de utilidad corresponde a la sesión 5. En contraste, los niveles más bajos se obtuvieron en las sesiones 6 en satisfacción, 1 en aprendizaje y 1 en utilidad.

Estas evaluaciones indican la apreciación subjetiva respecto a la utilidad, satisfacción y aprendizaje de los participantes respecto a las sesiones.

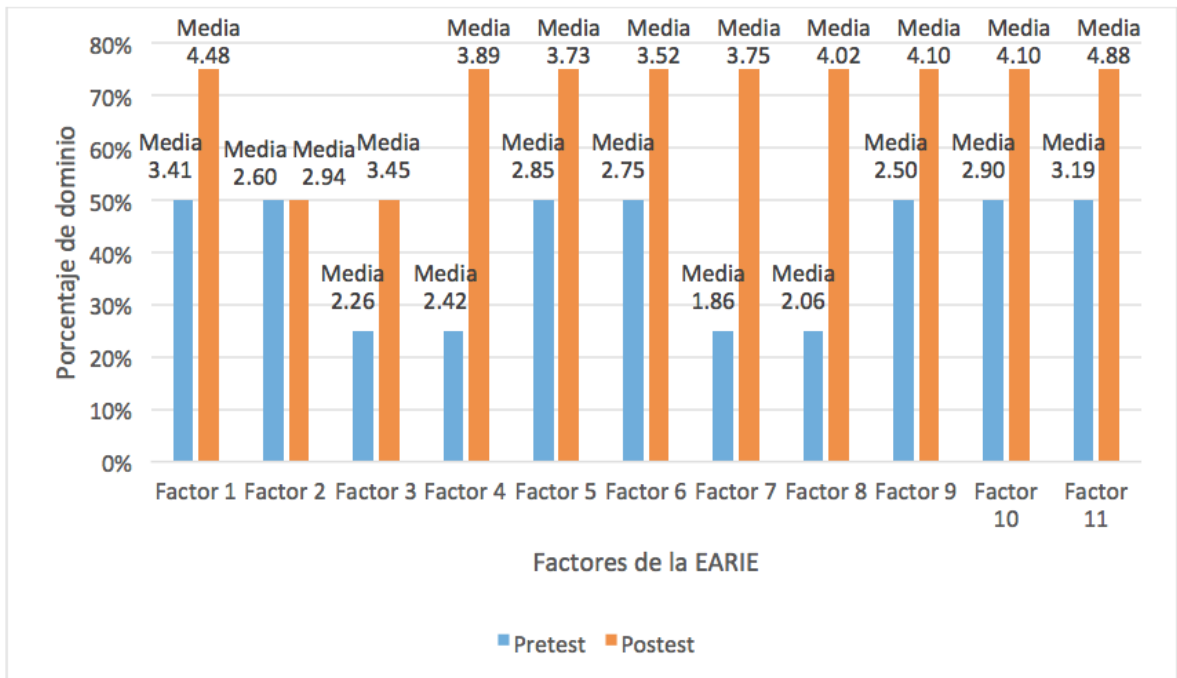

Figura 1. Comparativo de los resultados obtenidos pretest y postest de la escala EAIE

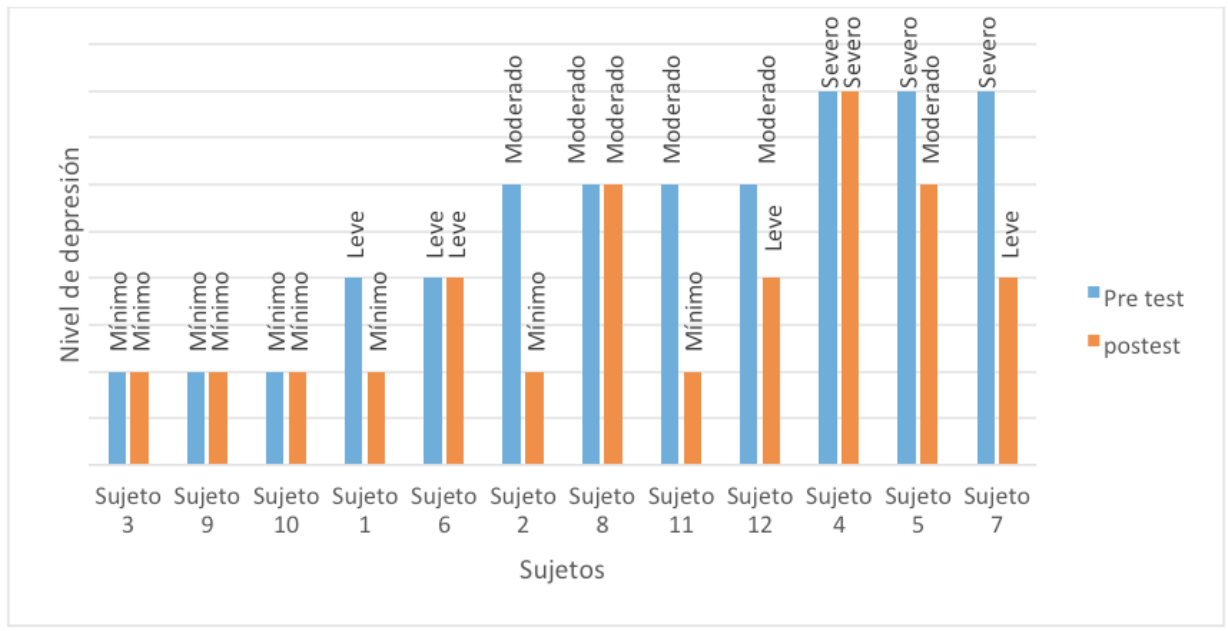

Figura 2. Gráfica Inventario de Depresión de Beck por sujeto en pretest y postest 


\section{Pruebas de hipótesis del efecto del taller}

Se llevó a cabo una prueba de hipótesis donde se determinó el efecto que tuvo el taller en las variables medidas en los participantes. Se probó la hipótesis nula de que no existirían diferencias estadísticamente significativas en las variables observadas antes y después de la aplicación del taller, versus la hipótesis alterna de que existirían mejoras estadísticamente significativas en las variables observadas antes y después del taller. Para probar estas hipótesis y dado el tamaño reducido de la muestra $(n=12)$, se utilizó la prueba de Rango con Signo de Wilcoxon en vista de que el diseño del estudio era un pretest/postest. En la tabla 4, se pueden observar los resultados obtenidos.

Tabla 4.

Resultados de la prueba de Rango con Signo de Wilcoxon

\begin{tabular}{lcccccc}
\hline \multirow{2}{*}{ Escala } & \multicolumn{2}{c}{ Pretest } & \multicolumn{2}{c}{ Postest } & \\
\cline { 2 - 5 } & Media & $\begin{array}{c}\text { Desviación } \\
\text { estándar }\end{array}$ & Media & $\begin{array}{c}\text { Desviación } \\
\text { estándar }\end{array}$ & Z & r \\
\hline BDI-II & 22.83 & 12.014 & 12.8333 & 11.14233 & $\mathbf{- 2 . 1 2 *}$ & $\mathbf{. 6 1}$ \\
EARIE & & & & & & \\
EAIE Factor 1 & 3.41 & .86 & 4.48 & .36 & $\mathbf{- 2 . 9 3 * *}$ & $\mathbf{. 8 5}$ \\
EAIE Factor 2 & 2.60 & .60 & 2.94 & .90 & $\mathbf{- 1 . 1 0}$ & .32 \\
EAIE Factor 3 & 2.26 & .83 & 3.45 & .72 & $\mathbf{- 2 . 5 9 * *}$ & $\mathbf{. 7 5}$ \\
EAIE Factor 4 & 2.42 & .82 & 3.89 & .73 & $\mathbf{- 3 . 0 6 * *}$ & $\mathbf{. 8 8}$ \\
EAIE Factor 5 & 2.85 & .67 & 3.73 & .63 & $\mathbf{- 2 . 5 9 * *}$ & $\mathbf{. 7 5}$ \\
EAIE Factor 6 & 2.75 & .96 & 3.52 & .75 & $\mathbf{- 1 . 6 4}$ & .47 \\
EAIE Factor 7 & 1.86 & 1.15 & 3.75 & .88 & $\mathbf{- 2 . 5 9 * *}$ & $\mathbf{. 7 5}$ \\
EAIE Factor 8 & 2.05 & .76 & 4.02 & .57 & $\mathbf{- 2 . 9 8 * *}$ & $\mathbf{. 8 6}$ \\
EAIE Factor 9 & 2.50 & .60 & 4.10 & .58 & $\mathbf{- 3 . 0 6 * *}$ & $\mathbf{. 8 8}$ \\
EAIE Factor 10 & 2.89 & 1.07 & 4.10 & .81 & $\mathbf{- 2 . 8 5 * *}$ & $\mathbf{. 8 2}$ \\
EAIE Factor 11 & 3.18 & 1.05 & 3.87 & 1.09 & $\mathbf{- 1 . 3 8}$ & .40 \\
\hline
\end{tabular}

${ }^{*} p<0.05 ;{ }^{* *} p<0.01 ;{ }^{* *} p<0.001$

Valores de $\mathrm{r}$.10 reflejan un tamaño del efecto trivial, de .10 a .299 Pequeño, de .30 a .499 Moderado, de .50 a .699 Grande y mayor o igual que .70 Muy Grande. Se interpretaría como el tamaño del efecto de la intervención sobre la variable comparada 
Como se puede apreciar en la tabla 4, el taller tuvo efecto en los síntomas de la depresión, y en IE de los participantes. En los factores 1, 3, 4, 5, 7, 8, 9 y 10 de la EAIE presentaron diferencias estadísticamente significativas y un efecto de aumento catalogado como muy grande. Es decir, hubo un incremento en las habilidades del darse cuenta emocional, tanto en el área intrapersonal como en el área interpersonal (1); predecir el cambio emocional, tanto en el área intrapersonal como en el área interpersonal (3); conocer las causas de las emociones y de la expresión emocional en el área interpersonal (4); controlar la distancia emocional en el área intrapersonal (5); conocer la expresión emocional en el área intrapersonal (7) y explicar las sensaciones de las emociones, tanto en el área intrapersonal como en el área interpersonal (8): reconocer el cambio emocional tanto en el área intrapersonal como en el área interpersonal (9); recordar emociones para acordarse de situaciones, tanto en el área intrapersonal como en el área interpersonal (10); y lograr emociones determinadas en el área intrapersonal (11).

También, se presentaron diferencias significativas en la escala de BDI-II al disminuir los niveles de depresión con una disminución considerada como Grande en las puntuaciones de depresión.

\section{Discusión}

En función a los resultados obtenidos en la prueba de Rango con Signo de Wilcoxón de las escalas de EAIE y BDI-II, hubo mejoras estadísticamente significativas, es decir, el taller tuvo efecto en cuanto a la sintomatología depresiva, e IE de los participantes.

En cuanto a los resultados de la EAIE, se observa una diferencia entre la evaluación pretest y la postest, en los factores cuatro (conocer las causas de las emociones y de la expresión emocional en el área interpersonal); siete (conocer la expresión emocional en el área intrapersonal); y ocho (explicar la sensación de las emociones tanto en el área intrapersonal como en el área interpersonal), que se refieren específicamente a la primera habilidad de Mayer y Salovey (1997), percepción, evaluación y expresión emocional, respectivamente, hubo una mejora significativa, esto puede ser debido a que durante el taller, se abordó dicha habilidad desde la primera sesión y desde ahí fue la base para la realización de las siguientes sesiones hasta la última, cuando las actividades iban dirigidas a ejercitar la habilidad para identificar la emoción en los estados físicos, fisiológicos y pensamientos de uno, así como expresar adecuadamente las emociones y las necesidades relacionadas con esos sentimientos y la habilidad para discriminar entre expresiones honestas y deshonestas (Mayer, \& Salovey, 1997).

Los participantes reportaron durante la intervención y en las tareas asignadas, la capacidad de poder identificar sus propias emociones y las de los demás, así como pudieron expresar de una manera adecuada sus sentimientos hacia las personas con quienes conviven, al sentir una 
mejoría en su bienestar emocional. Esto se demuestra, ya que al manejar de manera adecuada la primera habilidad de percepción y expresión emocional, a la cual pertenecen dicho factores, donde hubo un incremento considerable, hay una mayor adaptación para hacer frente a las demandas sociales y retos personales; existe una mayor competencia social y, por tanto, mayor red social; mejor manejo del estrés y de los conflictos (Extremera, \& Ruiz, 2012).

En cuanto al factor tres (predecir el cambio emocional tanto en el área intrapersonal como en el área interpersonal); el factor cinco (controlar la distancia emocional en el área intrapersonal); el factor nueve (reconocer el cambio emocional tanto en el área intrapersonal como en el área interpersonal) y el factor 10 (recordar emociones para acordarse de situaciones, tanto en el área intrapersonal como en el área interpersonal), pertenecientes a la segunda habilidad que implica la utilización del conocimiento emocional en lo intrapersonal e interpersonal, además de la cuarta habilidad que implica la regulación reflexiva de las emociones intrapersonal. También hubo un incremento significativo en dichos factores, este incremento puede estar relacionado con que en el taller se abordaron temas con respecto a dichas habilidades, se les enseñaba que las emociones son tan intensas que pueden ser generadas como ayuda del juicio y de la memoria sobre los sentimientos y que el humor puede cambiar la perspectiva del individuo desde el optimismo al pesimismo, favoreciendo la consideración de múltiples puntos de vista
(Mayer, \& Salovey, 1997). De igual manera, los participantes aprendieron a tolerar las emociones tanto agradables como desagradables y a saber disminuir aquellas emociones que no les gusta o aumentar las emociones agradables.

Aunque no hubo una diferencia estadísticamente significativa, el factor seis (controlar la distancia emocional en el área interpersonal) y el factor 11 (lograr emociones determinadas en el área intrapersonal), pertenecientes a la cuarta habilidad regulación de las emociones, se observa un ligero aumento en las medias, quizás se deba a que el tiempo dedicado a dichos factores no fuera suficiente, ya que al ser las habilidades más complejas se les dedicaron dos sesiones, a diferencia de la primera y segunda habilidad, en donde, durante todas las sesiones se abordaban con ejemplos y actividades.

Al ser temas que los participantes reportaron que desconocían al momento de enseñarles las habilidades, probablemente el ligero incremento pudo deberse a la capacidad cognitiva, característica de los jóvenes que se encuentran en la etapa cuando la la memoria goza de mayor agudeza y flexibilidad frente a las diversas situaciones a las que enfrenta (Papalia et al., 2009). Si bien, como menciona Mestre y FernándezBerrocal (2009), el conocimiento emocional comienza desde la infancia y crece a lo largo de toda la vida, con una comprensión aumentada de los significados emocionales, y conforme se avanza el desarrollo de una persona, ésta va progresando en el razonamiento sobre las secuencias de las emociones. 
En relación con los resultados comparativos del pretest y del postest, arrojados en el BDIII, se encontraron diferencias significativas, es decir, hubo un decremento en los puntajes, al observarse que en los participantes, la mayoría de ellos disminuyó de nivel, excepto uno que se mantuvo en nivel severo, puede ser debido a que a la semana de finalizar el taller, el participante reportó haber sufrido una pérdida importante y de gran significado para él, además de un participante que se mantuvo en nivel moderado. Este decremento de la mayoría de los participantes, se deba quizás en función a los factores cuatro, siete y ocho que se refieren a la primera habilidad de IE de percepción, evaluación y expresión emocional, que son los que aumentaron considerablemente y tuvieron mejoras significativas en la EAIE.

Tal como se refleja en la literatura, aquellos participantes que tienen mayor percepción para identificar y reconocer sus emociones en lo fisiológico y cognitivo, se permiten disminuir la intensidad de los estados de ánimo negativos provocados por los acontecimientos adversos a la vida (Fernández-Berrocal, \& Extremera, 2006). También, se puede explicar esta disminución en las puntuaciones de depresión, ya que se intervino en el cambio de creencias des-adaptativas, lo cual concuerda con Granado, Gomila y Filella (2013), así como con Rodríguez, Sosa-Correa, García y Bozal (2017), en cuanto a que el cambio a cogniciones adaptativas influye en la generación de emociones igualmente adaptativas.
Durante el taller, los participantes fueron realizando actividades en función a la exploración de conocerse a sí mismos, conocer sus emociones y pensamientos, mediante autorregistros y evaluaciones emocionales. Este punto a considerar en la realización de actividades de autoaceptación, permitió que reconocieran sus propios emociones; al no evaluarse como bueno o malo, sino en función de sus pensamientos, emociones, conductas y aceptarse incondicionalmente a dichas características, se tiene mejor manejo de sus emociones no adaptativas y una disminución en la depresión (Ellis, 2000).

Entre las limitaciones de este estudio se encuentra el bajo número de sujetos que participaron en el pretest, la intervención y el postest; no se contó con un grupo control, el cual daría más relevancia a los datos encontrados. Además, el instrumento utilizado para medir al IE estima la percepción, pero no la capacidad.

Para los fines de la investigación, se concluye que la intervención propuesta tuvo un impacto significativo en el incremento en los puntajes de las escalas aplicadas a los universitarios con síntomas depresivos, al aumentar los niveles obtenidos en las pruebas de IE y en la disminución de los niveles de depresión, de acuerdo con los objetivos propuestos.

\section{Referencias}

Amérigo, M. (1995). Satisfacción Residencial. Un análisis psicológico de la vivienda y su entorno. Madrid: Alianza editorial. 
Aragonés, J. I., \& Amérigo, M. (2000). Psicología Ambiental. Aspectos conceptuales y metodológicos. En J. Aragonés \& M. Amérigo (Coor.), Psicología Ambiental. Madrid: Pirámide.

Baron, R. (1972). Aggression as a function of ambient temperature and prior anger arousal. Journal of Personality and Social Psychology, 21, 183-189.

Baron, R. (1978). Aggression and heat: The "long hot summer". En A. Baum, S. Valins \& J. E. Singer (Eds.), Advances in Environmental Research (pp. 186-207). Hillsdale, NJ: Lawrence Erlbaum Associates Inc.

Bell, P. A., \& Greene, T. C. (1982). Thermal stress: Physiological, comfort, performance, and social effects of hot and cold environments. En G. E. Evans (Ed.), Environmental Stress (75-104). EUA: Cambridge University Press.

Bell, P. A., \& Fusco, M. E. (1989). Heat and violence in the Dallas field data: Linearity, curvilinearity and heteroscedasticity. Journal of Applied Social Psychology, 19, 1979-1982.

Bentler, P. M. (2006). EQS Structural Equations Program Manual Multivariate Software. California: Encino.

Cassidy, T. (1997). Environmental Psychology. Behavior and experience in context. Birmingham, UK: Psychology Press.

Cohen-Sánchez, L. (2009). Efectos de la intensidad de la luz en el ser humano y su expresión a través del color de la vestimenta. Anales Universitarios de Etología, 3, 29-35.
Cohn, E. G. (1993). The prediction of police call for service: The influence of weather and temporals variables on rape and domestic violence. Journal of environmental psychology, 13, 71-83.

Corral, V. (2010). Psicología de la sustentabilidad, un análisis de lo que nos hace pro ecológicos y pro sociales. México: Trillas.

Cuervo, N. (2012). Los avatares de la vivienda en la investigación urbana y regional latinoamericana (1990-2012). Territorios, 27, 47-70.

Díaz, D., Rodríguez-Carvajal, R., Blanco, A., Moreno-Jiménez, B., Gallardo, I., Valle, C., \& van Dierendonck, D. (2006). Adaptación española de las escalas de bienestar psicológico de Ryff. Psicothema, 18, 572-577.

Field, S. (1992). The effect on temperature on crime. British Journal of Criminology, 32, 340-351.

García-Viniegras, C. (2005). El bienestar psicológico: Dimensión subjetiva de la calidad de vida. Revista Electrónica de Psicología Iztacala, 8, 1-20.

Hernández, B. G., \& Gómez A. A. (2007). La temperatura ambiental y su vinculación con el aprovechamiento escolar. Palapa, Revista de Investigación Científica en Arquitectura, 2, 21-30.

Holahan, C. J. (2010). Psicología ambiental un enfoque general. México: Limusa.

Keller, M. C., Fredrickson, B. L., Ybarra, O., Cote, S., Johnson, K., Mikels, J., \& Wager, T. (2005). A warm heart and clear head: The 
contingent effects of weather on mood and cognition. Psychological Science, 16, 724731.

Lambert, G., Reid, C., Kaye, D., Jennings, G., \& Esler, M. (2003). Increased Suicide Rate in the Middle-Aged and Its Association With Hours of Sunlight. The American Journal of Psychiatry, 160, 793-795.

Lewin, K. (1964). Field Theory in Social Science: Select theoretical papers. New York: Harper and Row.

Lionel, C. L. \& Olivera, S. M. (2013). Evaluación económica de la resistencia térmica de la vivienda de interés social en las ciudades tropicales de México. Acta Universitaria de la Universidad de Guanajuato, 23, 17-29.

Lotito, C. F. (2009). Arquitectura, psicología, espacio e individuo. Aus (Valdivia), 6, 12-17. ISSN 0718-7262.

Ponce, G. (2011). Habitar en México: Calidad y rezago habitacional en la primera década del milenio [Publicado en versión preliminar del documento de trabajo No. 112 del Centro de Estudios Sociales y de Opinión Pública de la Cámara de Diputados, LX Legislatura]. México.

Rim, Y. (1975). Psychological test performance of different personality types on Sharav days in artificial air ionization. International Journal of Biometeorology, 21, 337-340.

Rosen, S. (1985). The weather: Wendy and grounchy. The Catholic Digest, 94-97.
Rosenthal, N. E. (1993). Winter blues. New York: Guilford Press.

Sánchez, C. J. (1998). Escala de Bienestar Psicológico. Madrid: TEA Ediciones.

Recibido: 26/08/2018

Revisado: 07/10/2018

Aceptado: 12/11/2018 